\title{
Chinas Industriepolitik: auf dem Wege zu einem neuen Erfolgsmodell?
}

\author{
Chinas Aufstieg zur zweitgrößten Wirtschaftsmacht und „Werkbank der Welt“ ist eng mit \\ seiner aktiven Industriepolitik verbunden. Nach drei Dekaden zweistelligen Wachstums \\ und einer deutlichen Verbesserung des Lebensstandards der Bevölkerung ist das chinesi- \\ sche Wirtschaftsmodell jedoch an seine Grenzen gestoßen. Eine stärker nachhaltige, \\ binnenmarktorientierte, sozial ausgeglichene und innovationsgetriebene Entwicklung ist \\ jetzt das Ziel. Um die Umsteuerung zu erreichen, nutzt die Regierung vielfältige industrie- \\ politische Instrumente und setzt auf strategische Industrien mit hoher technologischer \\ Wettbewerbsfähigkeit. Vor allem für Länder mit nachholender Entwicklung sind die \\ industriepolitischen Erfahrungen Chinas von Bedeutung.
}

MARGOT SCHÜLLER

\section{Besonderheiten der chinesischen Industriepolitik}

Dass China heute die zweitgrößte Wirtschaftsmacht, das weltweit größte Exportland und der wichtigste Standort für das verarbeitende Gewerbe ist, hängt eng mit seiner Industriepolitik und der proaktiven Rolle des Staates zusammen, die das Selbstverständnis der politischen Führung prägt. Der Staat soll die Entwicklung bestimmter Industrien fördern und selbst als Unternehmer über staatliches Eigentum an Unternehmen eine Vorreiterrolle spielen. Diese Vorstellung ist konträr zur Diskussion um Legitimität und Effizienz der Industriepolitik im Westen. Dort soll sich der Staat aus Sicht der Neoklassik im Wesentlichen auf die Schaffung unternehmensfreundlicher Rahmenbedingungen beschränken. Der rasante wirtschaftliche Aufstieg einer Reihe ostasiatischer Länder wie Japan, Südkorea und Taiwan, die zeitversetzt über lange Entwicklungsperioden hohe Wachstumsraten verzeichnen konnten, hat jedoch zu einer Neubewertung der Rolle des Staates geführt. Dieser wurde als „Entwicklungsstaat“ (developmental state) von beispielsweise Johnson (1982), Amsden (1989) und Wade (1990) beschrieben, der durch eine aktive Intervention den wirtschaftlichen Aufholprozess erleichtert und den erfolgreichen Strukturwandel fördert. Dass auch China ab Anfang der 1980er Jahre als Entwicklungsstaat eingestuft werden kann, erklärt Knight (2012, S. 5f.) mit der besonderen Motivati- onsstruktur der politischen Elite. Während in Japan und Korea die Herausbildung eines Entwicklungsstaates als Reaktion auf Krisen und äußere Bedrohung entstand, musste die Kommunistische Partei Chinas (KPCh) nach dem Tode Mao Zedongs ihren Fokus auf ein hohes Wirtschaftswachstum in Verbindung mit einer Verbesserung des Lebensstandards legen, um die verlorene Legitimität in der Bevölkerung wiederherzustellen.

Während die chinesische Regierung nach wie vor eine proaktive Wirtschaftspolitik verfolgt, hat sich die Diskussion um staatliches Eigentum an Unternehmen verstärkt. Dies betrifft vor allem die weiterhin in Schlüsselpositionen operierenden großen Staatsunternehmen. Charakteristisch für die Industriepolitik Chinas ist ferner die starke Rolle der Lokalregierungen, die eigene Verfügungsrechte an Staatsunternehmen halten und unabhängige Industriestrukturen aufweisen. Die Fragmentierung führt zu Überlappungen und Interessengegensätzen zwischen Zentrale und Lokalregierungen. Dies erschwert eine Umsetzung industriepolitischer Leitlinien der Zentralregierung und begünstigt lokale Varianten. Andererseits waren räumlich begrenzte Reformexperimente - entweder zentralstaatlich vorgegeben oder im Nachhinein akzeptiert - ein wichtiges Politikelement. Dazu zählten beispielsweise die Anfang der 1980er Jahre gegründeten Sonderwirtschaftszonen (SWZ), die steuerliche Anreize und marktwirtschaftliche Rahmenbedingungen für die exportorientierte Produktion und für ausländische Direktinvestitionen boten. 
Im Vergleich zu westlichen Industriestaaten sind die politischen und wirtschaftlichen Akteure in China auch auf andere Weise miteinander verflochten. Die industriepolitisch relevanten Institutionen wie Zentralbank, Geschäftsbanken, Staatsunternehmen, Gewerkschaften, Verbände, Gerichte und Regulierungsbehörden haben keine Entscheidungsautonomie, sondern sind über mittel- und langfristige Entwicklungspläne, Mitgliedschaft in der KPCh und über Regierungsorganisationen verschiedener Ebenen miteinander verbunden bzw. voneinander abhängig und damit Teil einer spezifischen Ordnung. Dies „chinesische Modell“ setzt auf einen speziellen Mix von Teilordnungen, die nicht mit dem westlichen Verständnis der Interdependenz und Komplementarität von politischer und wirtschaftlicher Ordnung kompatibel sind. Hierbei geht es nicht nur um die grundsätzliche Frage, ob Marktwirtschaften in einem autoritären politischen System erfolgreich sein können, sondern welche ordnungspolitischen Elemente die Funktionsfähigkeit marktwirtschaftlicher Strukturen unterstützen.

Die Frage der Komplementarität von Institutionen steht auch im Mittelpunkt des Varieties of Capitalism (VoC)Ansatzes, mit dem ebenfalls versucht wurde, die chinesische Modellvariante zu erklären. Hierbei sind die Unternehmen Hauptakteure des institutionellen Wandels und ihre Beziehungen zum Finanzsektor, zur (Berufs-)Bildung, zu den Beschäftigten, zur Wettbewerbsordnung und zum Wohlfahrtssystem werden untersucht (Hall/Soskice 2001; Hall/Gingerich 2004). Allerdings lässt sich mit diesem Ansatz das Zusammenspiel der Institutionen in China auch nur in beschränktem Maße erklären. Denn es gibt in diesem Ansatz nur zwei Kategorien, nämlich, den liberal-unkoor- dinierten Kapitalismus (liberal market economy/LME) und den koordinierten Kapitalismus (coordinated market economy/CME) bzw. die Spielart „Asian Capitalism“ (AC). In China sind jedoch beispielsweise die Beziehungen zwischen Unternehmen und Beschäftigten dadurch geprägt, dass einerseits Ähnlichkeiten zu den CMEs bestehen, da es formal (gesetzlich festgeschrieben) einen starken Arbeitnehmerschutz gibt. Andererseits ist der Arbeitnehmerschutz de facto schwach und die Arbeitsbeziehungen zwischen Unternehmen und Beschäftigten vergleichbar strukturiert wie in den LMEs. Im chinesischen Modell ist der Einfluss des politischen Systems die entscheidende zusätzliche Variable. Diese erklärt, dass Gewerkschaften zwar auch in China existieren, aber nicht unabhängig sind und dass beispielsweise der Vorsitzende des chinesischen Gewerkschaftsbundes als Mitglied des Politbüros der KPCh einen höheren Status als der Arbeits- und Sozialminister hat.

\section{Strategien und Programme bis 2008}

Die chinesische Industriepolitik lässt sich in verschiedene Phasen einteilen (Übersicht 1). Bis Ende der 1970er Jahre dominierten die Förderung der schwerindustriellen Entwicklung, eine Importsubstitutionspolitik, Staatsunternehmen sowie die staatliche Planungsbürokratie. Das Industriewachstum fiel mit real durchschnittlich 10,5\% zwischen $1952-1978$ zwar hoch aus, doch der Lebensstandard

ÜBERSICHT 1

\section{Chinas industriepolitische Schwerpunkte}

\begin{tabular}{lll}
\hline Jahre & Ziele, Politiken und Programme & Schwerpunkte der staatlichen Förderung \\
\hline $1950-1970$ & $\begin{array}{l}\text { Industrialisierung, Importsubstitution, } \\
\text { Binnenmarktorientierung }\end{array}$ & Schwerindustrie \\
\hline $1980-1990$ & Reformexperimente, Sonderwirtschaftszonen (SWZ) & $\begin{array}{l}\text { Maschinenbau, Energieausrüstungen, Automobil- und } \\
\text { Luftfahrtindustrie }\end{array}$ \\
\hline $1990-2000$ & $\begin{array}{l}\text { Sozialistische Marktwirtschaft } \\
\text { Restrukturierung der Staatsunternehmen } \\
\text { Anreize für Auslandsinvestitionen } \\
\text { Industriepolitik für Automobilindustrie }\end{array}$ & $\begin{array}{l}\text { Automobilindustrie, Elektronik, Baustoffsektor, } \\
\text { Maschinenbau, Petrochemie }\end{array}$ \\
\hline $2000-2010$ & $\begin{array}{l}\text { WTO-Beitritt und Politikanpassung, Programm } \\
\text { für eigenständige Innovation; Förderung der } \\
\text { Internationalisierung chinesischer Unternehmen, } \\
\text { Restrukturierung der Industrie, Konjunkturprogramm } \\
\text { ab 2008 (Auswirkungen der globalen Finanzmarktkrise) }\end{array}$ & $\begin{array}{l}\text { Kutomatisierung. } \\
\text { industrie, Textil- und Maschinenbauindustrie, } \\
\text { Elektronik, IT-Industrie, Petrochemie }\end{array}$ \\
\hline $2010-2015$ & $\begin{array}{l}\text { Restrukturierung der Industrie: höhere Wertschöpfung } \\
\text { Energieeffizienz } \\
\text { Revitalisierung der traditionellen Industrien }\end{array}$ & $\begin{array}{l}\text { IT-Industrie, Logistik, strategische Industrien: } \\
\text { energieeffiziente und umweltfreundliche Techno- } \\
\text { logien, Biotechnologie, neue Materialien, Luft- und } \\
\text { Raumfahrtausrüstungen, Satellitentechnologie, }\end{array}$ \\
\hline
\end{tabular}


verbesserte sich kaum. ${ }^{1}$ Mit der schrittweisen Transformation des Wirtschaftssystems ab Anfang der 1980er Jahre wurden sozialistische Entwicklungsziele und Politiken durch eine stärker marktwirtschaftliche Orientierung und außenwirtschaftliche Öffnung ergänzt. Neben Elementen der horizontalen Steuerung wie fiskalischen und kreditpolitischen Anreizen blieb die vertikale Steuerung in Form beispielsweise der Förderung bestimmter Industrien bestehen. Diese Dualität verschiedener Steuerungselemente kennzeichnet auch den Unternehmenssektor. So wurden zwar private und ausländische Unternehmen zugelassen, doch Staatsunternehmen blieben als „Rückgrat“ der Industrieentwicklung erhalten. Sie wurden ab Mitte der 1980er Jahre rechtlich umstrukturiert, teilweise zu Unternehmensgruppen fusioniert, kleinere Staatsunternehmen auf lokaler Ebene weitgehend privatisiert und große Staatsunternehmen einer staatlichen Treuhandgesellschaft (der Stateowned Assets Supervision and Administration of the State Council, SASAC) unterstellt.

Vergleichbare industriepolitische Leitlinien und branchenbezogene Programme wie in den asiatischen Nachbarländern veröffentlichte die chinesische Regierung erst Mitte der 1990er Jahre. Während die Anzahl der Programme zunächst auf wenige pro Jahr beschränkt blieb, stieg ihre Zahl im Zuge der globalen Finanzmarktkrise nach 2008 schnell an (Heilmann/Shih 2013, S. 3). Als Schlüsselsektoren wurden in den 1980er und 1990er Jahren zunächst der Maschinenbau und Baustoffsektor, die Elektronik- und Automobilindustrie sowie die petrochemische Industrie ausgewählt und ihre Entwicklung über Steuer- und Zinserleichterungen gefördert und durch Zollbarrieren vor ausländischer Konkurrenz geschützt. Zur Förderung der industriellen Schlüsselsektoren lenkte die Regierung mit Hilfe eines Investitionskatalogs Auslandsinvestitionen und Know-how in diese Industrien. Auch mit dieser Politik folgte China den Strategien des ,industrial targeting" und „picking winners", die zuvor in Japan und Südkorea eingesetzt worden waren, um Struktur und Entwicklung der Industrie zu beeinflussen. Im Unterschied zu diesen Ländern war das technologische Entwicklungsniveau Chinas jedoch weitaus niedriger zum Zeitpunkt der außenwirtschaftlichen Öffnung des Landes, sodass den Auslandsinvestitionen ein größeres Gewicht eingeräumt wurde. Über die Integration chinesischer Unternehmen in die Wertschöpfungsketten ausländischer Hersteller erfolgte der Transfer von technischem und Management Know-how. Hierdurch verbesserte sich die Wettbewerbsfähigkeit chinesischer Unternehmen auf den In- und Auslandsmärkten. Obwohl die chinesische Regierung mit ihrem Beitritt zur World Trade Organization (WTO) Ende 2001 den ausländischen Investoren eine Gleichbehandlung zusicherte, besteht diese bis heute nur eingeschränkt. Nach wie vor existieren Zutrittsbarrieren in den Branchen, in denen die chinesischen Unternehmen noch nicht ausreichend wettbewerbsfähig sind. Im Markt werden ausländische Unternehmen stärker diskriminiert als inländische, beispielsweise bei der Vergabe von Staatsaufträgen (Covington/Burling 2014).
Im Rahmen seiner Industriepolitik setzte China im technologischen Aufholprozess nicht nur auf den Technologietransfer aus dem Ausland, sondern auch auf den Ausbau der eigenen Wissenschafts- und Technologiebasis. Zu den verschiedenen Programmen des Wissenschafts- und Technologieministeriums (Ministry of Science and Technology/ MOST) gehört das „High Tech Research and Development“Programm, das im März 1986 begann und eine Laufzeit von 15 Jahren hatte. Dies sogenannte „863“--Programm förderte vor allem die Wissenschafts- und Technologieentwicklung im Agrarsektor, traditionelle Industrien und Hightech-Industrien. Im Einzelnen zählten dazu Agro-Biotechnologie und medizinische Biotechnologie, Verarbeitungstechnologien, Entwicklung von Kernreaktoren und die militärische Forschung. Strategisch wichtige Hightech-Felder waren Automatisierung, Biotechnologie, Raumfahrt-, Informations-, Laser-, Energie-, Meeres- und Materialtechnologie. Das 863-Programm umfasste mit sieben Schwerpunktbereichen ein Fördervolumen von 600 Mio. € für den Zeitraum 1986 bis 2000. Das Programm wurde in den anschließenden Jahren fortgesetzt und für den Zeitraum 2001 - 2005 mit einem erhöhten Budget in Höhe von 1,5 Mrd. € ausgestattet (Schüller 2008, S. 55f.).

Das MOST fördert auch die 160 nationalen Forschungsinstitute, internationale Programme wie das Galileo-Sattelitenprogramm, das „973“-Programm für Megaprojekte der Grundlagen- und der angewandten Forschung sowie Unternehmensneugründungen in Industrieparks im Rahmen des „Fackel“-Programms. Dies setzt u.a. auf die Gründung von „High Technology Development Zones“, die entweder der Zentralregierung oder den Lokalregierungen unterstehen. Der Zhongguancun-Technologiepark im Bezirk Haidian in Beijing stellt das national bedeutendste F\&E-Zentrum nach dem Vorbild des Silicon Valley in den USA dar (Walsh 2003, S. 45; Schüller/Conlé 2007, S. 65). Die Gründung von Universitäts- und Technologieparks zielt vor allem auf im Ausland befindliche chinesische Wissenschaftler, die nach China zurückkehren sollen. Um diesen „reverse brain drain“ zu fördern, legte die Regierung verschiedene Programme für Rückkehrer auf (Wogart/Schüller 2011). Als Querschnittstechnologien förderte die Regierung vor allem Informations- und Kommunikationstechnologien (IKT), die zur Technologiediffusion innerhalb des Landes beitragen sollten. Hierfür wurde beispielsweise die Infrastruktur für Telekommunikations- und Internettechnologien ausgebaut, vor allem durch Glasfaserverkabelung und Mobilfunknetze (Naughton 2007, S. 370f.).

Die chinesische Industriepolitik stand nach dem WTOBeitritt Ende 2001 vor einem erheblichen Anpassungszwang. So mussten die ausländische Investoren diskrimi-

1 Der reale Pro-Kopf-Konsum zwischen 1952-1978 der ländlichen Bevölkerung, die mit 80 \% den Hauptteil der Gesamt bevölkerung stellte, wies lediglich eine Wachstumsrate von $1 \%$ pro Jahr auf (Knight 2012, S. 4). 
nierenden industriepolitischen Bestimmungen wie „local content“-Auflagen (Festlegung eines im Inland hergestellten Produktionsanteils), Exportquoten und der Joint VentureZwang abgeschafft und Gesetze und Durchführungsbestimmungen an internationale Standards angepasst werden. Übergangsfristen haben diesen Anpassungsprozess erleichtert, der allerdings bis heute noch nicht völlig abgeschlossen ist. Mit dem WTO-Beitritt wurden eine stärkere Marktöffnung für ausländische Unternehmen und mehr Wettbewerb auf dem Inlandsmarkt erwartet. Vor diesem Hintergrund und um den Übergang von der arbeitsintensiven zur wissensintensiven Produktion zu fördern, legte die Regierung den Schwerpunkt der Technologiepolitik auf die eigenständige technologische Entwicklung. Das mittel- und langfristige Technologieentwicklungsprogramm (2006-2020) bildet den Rahmen für die neuen Ziele und Instrumente zum Aufbau eigenständiger Innovationskapazitäten. Parallel dazu begann die Regierung, die Internationalisierung chinesischer Unternehmen durch Investitionen im Ausland zu fördern, um den Zugang zu natürlichen Ressourcen und technologieintensiven Auslandsunternehmen zu sichern.

Allerdings konnte die Zentralregierung ihre industriepolitischen Vorstellungen nicht immer gegen den Widerstand lokaler Interessen durchsetzen. Dies zeigt das Beispiel der Automobilindustrie. ${ }^{2}$ So förderte die Zentralregierung anfänglich nur drei große und drei kleine Automobilwerke, deren Entwicklung durch hohe Zölle und andere Markteintrittsbarrieren vor ausländischer Konkurrenz geschützt wurde. Für diese Unternehmen wurden ausländische Joint Venture-Partner ausgewählt, die über Ausbildungsleistungen und Produktionstechnologien den Technologietransfer im Gegenzug zum Marktzugang erhielten. Die ausländischen Automobilkonzerne nahmen im „Huckepack“-Verfahren ihre eigenen Zulieferunternehmen mit nach China, die oftmals auch Joint Ventures mit chinesischen Partnern eingingen. Zwar hatte die chinesische Automobilindustrie ihr Ziel der Importsubstitution Mitte der 1990er Jahre erreicht, doch waren die Fahrzeuge sehr teuer, wiesen keine neuen technischen Entwicklungen auf und waren damit nicht international konkurrenzfähig. In dieser Situation entwickelte sich ein lokales Gegenmodell mit rein chinesischen Unternehmen. Die ersten zwei Unternehmen waren Chery Automobil Company aus der Stadt Wuhu, Anhui Provinz und Geely Automobile Company aus Taizhou in der Provinz Zhejiang. In beiden Unternehmen erfolgte der Aufbau mit Hilfe der Lokalregierungen: Chery war auf Initiative des KPCh-Parteisekretärs der Stadt gegründet worden, und auch Geely erhielt Unterstützung durch die Lokalregierung. Beide besaßen anfänglich keine Produktionslizenz der Zentralregierung, hatten jedoch die Provinzregierungen auf ihrer Seite, die hierfür Lobbyarbeit betrieben. Nachdem die Unternehmen sich erfolgreich entwickelten, stimmte die Zentralregierung diesen Experimenten zu und erklärte sie zu Modellen der „eigenständigen Innovation“. Sie erteilte zwei weiteren, rein chinesischen Unternehmen (Hafei und Brilliance) eine Montagelizenz, um den Wettbewerb weiter zu beleben. Ein neuer industriepolitischer Ansatz in der $\mathrm{Au}$ tomobilindustrie entstand, der auf dem Kauf von Lizenzen und technischen Ausrüstungen sowie auf Fachkräften basierte, die von sino-ausländischen Joint venture-Unternehmen abgeworben wurden und neue Fahrzeugdesigns entwarfen. Die Unternehmen fokussierten auf neue Käufergruppen, die preisgünstige Fahrzeuge bevorzugten, sie bezogen KfzTeile durch Zulieferketten im Inlandsmarkt und expandierten schnell im internationalen Markt (Schüller 2012; Chu 2011).

Weiterhin wurde die Umsetzung der Industriepolitik Heilmann und Shih (2013, S. 7ff.) zufolge durch die Konkurrenz verschiedener Ministerien erschwert. So überlappten sich beispielsweise in den 1990er Jahren die Kompetenzen der State Planning Commission (SPC) in der Industriepolitik, zuständig für die Erstellung von Entwicklungsplänen mit verschiedenen Laufzeiten, mit denen der State Economic Commission, die für die Umsetzung der Planvorgaben durch die Staatsunternehmen verantwortlich war, und mit den Vorschlägen zur Reform institutioneller Strukturen durch die State Structual Reform Commission. Administrative Reformen unter Ministerpräsident Zhu Rongji führten dazu, dass für die Industriepolitik neben der SPC die 1993 neu gegründete State Economic and Trade Commission (SETC) sowie das Development Research Center des Staatsrats zuständig wurden. SETC absorbierte die Ministerien für Kohle, Metallurgie und Maschinenbau, die sie in Behörden umwandelte, und trieb die Reform der Staatsunternehmen voran. Ein weiterer Zusammenschluss wurde im Jahr 2003 durchgeführt. Durch die Fusion der SETC mit der reformierten SCP entstand die National Development and Reform Commission (NDRC), die als „Super-Ministerium“ und Treiber einer koordinierten industriellen Restrukturierung nach dem Vorbild des japanischen MITI arbeiten sollte (ten Brink 2013, S. 71). Das Eigentum der Staatsunternehmen wurde der SASAC unterstellt, während für das Design der Industriepolitik vor allem die NDRC verantwortlich wurde. Dabei wird die Kommission durch das 2008 neu gegründete Ministry of Industry and Information Technology (MIIT) bei branchenübergreifenden Politiken unterstützt.

Trotz der oben diskutierten Schwächen hat die Industriepolitik zur Verbesserung der internationalen Wettbewerbsfähigkeit chinesischer Unternehmen beigetragen. Ohne den massiven Schutz neuer Industrien, staatlicher Subventionen und den Transfer von Technologien ausländischer Unternehmen in China wäre ein Überleben der chinesischen Industrie auch nach Einschätzung chinesischer Experten kaum möglich gewesen (Liu 2005, S. 165). An diesem Erfolg waren auch die Lokalregierungen beteiligt, die industriepolitische Maßnahmen aufgrund ihrer Nähe zu den Unternehmen besser als die Zentralregierung

2 Siehe zu dieser Diskussion Chu (2011) sowie Lee et al. (2009). 
anpassen konnten und somit viele Erfolgsbeispiele hervorbrachten

\section{Industriepolitische Antworten auf die globale Finanzmarktkrise}

Die Fokussierung auf ein hohes Wirtschaftswachstum katapultierte China zwar in die Spitze der größten Industriestaaten, doch hatte diese Entwicklung einen hohen Preis. Die negativen Auswirkungen auf Umwelt, Finanzsystem und Einkommensverteilung hatten eine Neuorientierung schon Ende der 1990er Jahre erforderlich gemacht. Die Diskussion über die notwendige Anpassung des Wirtschaftsmodells hin zu einer stärker binnenmarktorientierten, sozial ausgeglichenen und innovationsgetriebenen Entwicklung verstärkte sich mit dem politischen Führungswechsel zu Hu Jintao (Generalsekretär der KPCh) und Wen Jiaobao (Ministerpräsident) in den Jahren 2002 und 2003. Die Vorstellungen zur Restrukturierung fanden Eingang in den 11. Fünfjahresplan (2006-2010), wurden aber nicht bzw. nur unzureichend umgesetzt. Vor allem in den Bereichen Ausbau des privaten Konsums (als Anteil am Bruttoinlandsprodukt/BIP), energieeffizientes und ressourcenschonendes Wachstum, Steigerung der Innovationskraft der Unternehmen sowie gerechtere Einkommensverteilung wurden die Ziele des Fünfjahresplanes nicht erreicht.

Als Reaktion auf die negativen Folgen der globalen Finanzmarktkrise für das Wachstum ${ }^{3}$ und die Beschäftigung legte die Regierung 2009 ein umfangreiches Konjunkturprogramm auf. Im Mittelpunkt standen staatliche Investitionen in die Infrastruktur und die Ausweitung lokaler Produktionskapazitäten, während die übrigen Ziele des 11. Fünfjahresplanes wie Restrukturierung und ökologische Aspekte in den Hintergrund traten (Schucher 2011; Schüller 2009). Vom Konjunkturprogramm mit einem Volumen von 495 Mrd. $€$ entfielen 37,5 \% der Mittel auf Infrastrukturprojekte, $25 \%$ auf den Wiederaufbau der durch das Erdbeben von 2008 zerstörten Gebiete in der Provinz Sichuan, $19 \%$ auf zivile Projekte allgemein und in ländlichen Gebieten sowie 9,25\% auf Technologieförderung und andere industriepolitische Maßnahmen (Schüller 2009). Zu den zehn Industrien, deren Wettbewerbsfähigkeit für das Wirtschaftswachstum als besonders wichtig angesehen wurde und deshalb gefördert werden sollte, zählten die Automobil- und Stahlindustrie, der Schiffbau, die Textil- und Maschinenbauindustrie, Elektronik und Informationstechnologien, Konsumgüterindustrie, Petrochemie, die Industrie für Nichteisen (NE)-Metalle und Logistik. Die Förderung bestand aus steuerlichen Anreizen für Unternehmen in diesen Branchen wie Exportsteuerrückerstattung und Subventionen für den Kauf bestimmter Güter wie Automobile und Elektronikgeräte durch Konsumenten. Weiterhin wurden die Banken angewiesen, Kredite für die o.g. Indus- trien zur Verfügung zu stellen. Sie erhielten direkte Finanzierungsmittel für technologische Neuerungen und zur Entwicklung eigener Marken. Zum Förderprogramm gehörte auch die Vergabe von Staatsaufträgen, die Ausweitung von Exportkrediten und von Krediten für Investitionen im Ausland (Morrison 2009).

Waren in den Jahren vor der globalen Finanzmarktkrise aufgrund des Widerstands gegen industriepolitische Programme für einzelne Branchen lediglich die Automobilindustrie (2004 durch NDRC) und der Maschinenbau (2006 durch Staatsrat) über spezielle Programme gefördert worden, wurde ab 2009 eine Vielfalt sektorspezifischer und sektorübergreifender Industrieprogramme eingeführt. Hierzu gehörten Heilmann und Shih (2013, S. 13f.) zufolge das sektorübergreifende Programm zur industriellen Technologiepolitik (2009 durch MIIT vorgelegt), die Programme von 2010 und 2011 zur Beschleunigung und Förderung der strategischen Industrien (Staatsrat, Nationaler Volkskongress, KPCh) sowie das Programm zur Restrukturierung und Modernisierung der Industrie 2011-2015 (2011 durch Staatsrat). Als branchenbezogenes Programm wurde 2009 beispielsweise die Revitalisierung der neun traditionellen Sektoren vom Staatsrat beschlossen sowie die Förderprogramme für IT-Industrie und Logistik. Weiterhin legten 21 Ministerien Fünfjahresprogramme für die Entwicklung einzelner Industrien vor.

Wie die verschiedenen industriepolitischen Programme mit ihren Schwerpunkten auf Restrukturierung und Modernisierung Chinas Industrieentwicklung der letzten Dekade beeinflusst haben, ist unklar. Zwar spiegeln sie die aktive Rolle des Staates im Aufholprozess mit langfristigen Planvorstellungen wider, die industriepolitischen Vorbilder der Nachbarländer einbezogen. Sie trafen aber auf andere institutionelle Rahmenbedingungen im Finanzsystem und Unternehmenssektor. Denn während die Programme der Zentralregierung auf technologische Modernisierung und neue strategische Industrien setzten und die eigenständige Innovation finanziell förderten, flossen auf der lokalen Ebene aufgrund leicht zugänglicher Kredite des staatlichen Bankensystems Investitionen vor allem in den weiteren Ausbau vorhandener Produktionskapazitäten der Staatsunternehmen und in nicht-produktive Bereiche wie den Immobiliensektor. Dass sich Chinas Produktionskapazitäten insgesamt erhöhten und die Bedeutung des Landes als „Werkstatt der Welt“ weiter stieg, zeigen auch die UNIDO-Statistiken. So nahm Chinas Anteil an der globalen Wertschöpfung der verarbeitenden Industrie zwischen 2002 und 2012 von 35 auf $50 \%$ zu. Mit rd. 68,8 Mio. (formal) Beschäftigten im verarbeitenden Gewerbe stellte China im Jahr 2010 den größten Anteil der Beschäftigten weltweit mit 34,3\% (Vergleich dazu Deutschland: 3,1\%) (UNIDO 2013, S. 9, 41).

3 So ging das Wachstum in China von $10,6 \%$ im 1. Quarta 2008 auf 6,8 \% im 4. Quartal 2008 zurück (Schüller 2009). 
Während China durch den Ausbau der verarbeitenden Industrie Wachstum und Beschäftigung auf einem hohen Niveau halten konnte, verschärfte sich das Problem der Überkapazitäten in der Industrie. Dies hatte bereits vor dem Ausbruch der globalen Finanzmarktkrise bestanden und die Regierung veranlasst, ab 2007 die Kreditvergabe an bestimmte Industrien wie die Stahl- und Zementindustrie sowie den Immobiliensektor zu beschränken. Zur Bekämpfung der globalen Finanzmarktkrise lockerte die Regierung 2009 jedoch im Rahmen ihres ehrgeizigen Konjunkturprogrammes wieder den Zugang zu Bankkrediten, sodass sich das Problem der industriellen Überkapazitäten in den Folgejahren erneut einstellte (Schüller 2009). So waren nach Berichten der NDRC im ersten Halbjahr 2013 nur 78\% der Produktionskapazitäten ausgelastet und es wurde erwartet, dass aufgrund noch nicht abgeschlossener Infrastrukturprojekte ein weiterer Kapazitätsausbau stattfinden würde (China Daily 2013).

Trotz der verstärkten industriepolitischen Anstrengungen, die strategischen Industrien auszubauen, konnten bisher nur wenige zur internationalen Spitze aufschließen. Ein Beispiel ist die IKT-Industrie, vor allem in den Segmenten Computertechnik und Telekommunikation. Innerhalb der Gruppe der Hightech-Industrien in China stellt die IKTIndustrie (Güterproduktion) die meisten Unternehmen und weist die beste Performanz auf. Sie hatte den größten Anteil in Bezug auf den Umsatz des Kerngeschäfts aller HightechIndustrien (47,7\%), den höchsten Gewinnanteil (38,2\%), das höchste Exportvolumen (57,1 \%) und die meisten F\&EAusgaben (50,5\%) im Jahr 2012. Ähnlich wie andere Hightech-Industrien weist auch die IKT-Industrie eine relativ niedrige Wertschöpfung aufgrund hoher Zulieferungen von Zwischenprodukten und technischen Komponenten auf. Nach wie vor muss China Schlüsseltechnologien der Chipherstellung oder Smartphone-Prozessoren importieren (Schüller/Schüler-Zhou 2015).

\section{Industriepolitische Dimensionen des neuen Wirtschaftsmodells}

Die globale Finanzmarktkrise hat mit verhindert, dass die bereits im 11. Fünfjahresplan (2006-2010) angestrebte Neuausrichtung des Wirtschaftsmodells umgesetzt wurde. Sie stärkte stattdessen die Rolle der Staatsunternehmen, die über öffentliche Aufträge im Infrastruktursektor und den Ausbau ihrer Produktionskapazitäten mehr Einfluss als zuvor gewannen. Denn mit dem Einbruch von Wachstum und Beschäftigung trat 2008 erneut die Frage der Legitimität des politischen Systems in den Vordergrund. Die Refokussierung auf hohes Wachstum erschwerte auch die notwendige Restrukturierung im Industriesektor. Die neue politische Führung unter Xi Jinping und Li Keqiang, deren Positionierung durch das Zentralkomitee (ZK) der KPCh im November 2012 und durch den Nationalen Volkskongress (NVK) im Frühjahr
2013 bestätigt wurde, nahm jedoch einen zweiten Reformanlauf. Auf der ZK-Tagung der KPCh im November 2013 wurde ein 60-Punkte-Reformpaket beschlossen und fand im Rahmen der NVK-Tagung im Frühjahr 2014 Eingang in die Jahresplanung des NDRC. Aufgrund dieses langwierigen Prozesses ist vom ehrgeizigen Reformanspruch erst relativ wenig in konkrete Politik umgesetzt worden bzw. kann hinsichtlich der Auswirkungen bewertet werden.

Auslöser für die Umsteuerung auf ein nachhaltiges, sozial ausgeglichenes und innovatives Wachstum, das als Reformziel im 60-Punkte-Programm von 2013 vorgegeben wurde, ist vor allem die steigende Unzufriedenheit der Bevölkerung mit der Umwelt- und Einkommenspolitik. Diese Unzufriedenheit spiegelte sich auch in einer repräsentativen Meinungsumfrage im Frühjahr 2013 in China wider. So bewerteten $47 \%$ (2008: $31 \%$ ) der Bevölkerung die Luftverschmutzung und $40 \%$ (2008: $28 \%$ ) die Wasserverschmutzung als größte Probleme. Die wachsenden Einkommensunterschiede wurden ebenfalls in der Befragung kritisch bewertet. Mehr als die Hälfte ( $52 \%$ ) beurteilten den Unterschied zwischen arm und reich als zu groß (2008: $41 \%$ ) (PEW 2013). Die von der Regierung angekündigte Umsteuerung auf einen innovationsgetriebenen Wachstumspfad wurde auch aufgrund des demografischen Wandels notwendig. Der Rückgang der Bevölkerungsteile im erwerbsfähigen Alter fällt zusammen mit steigenden Lohnkosten und macht Produktivitätssteigerungen immer nötiger. Nur wenn bei höherer Wertschöpfung die Einkommen und der Lebensstandard der Bevölkerung weiter steigen, wird die „middle income trap“, also ein Verharren auf dem bisherigen Entwicklungsniveau, vermieden werden können.

In der Industriepolitik knüpfen die Beschlüsse der neuen politischen Führung an die Ziele im 12. Fünfjahresplan (2011 - 2015) an und bieten deshalb eine gewisse Kontinuität. Neben der Restrukturierung und Modernisierung traditioneller Industriezweige wie Anlagenbau, Schiffbau, Automobil- und Stahlindustrie, der Industrien für NE-Metalle, Baustoffe, Petrochemie und der Leicht- und Textilindustrie fokussiert der Plan auf die Förderung von sieben sich neu entwickelnden „emerging industries“. Hierzu zählen neueste (next generation) Informationstechnologien, Industrien, die Produkte im Zusammenhang mit der Verwendung von energieeffizienten und umweltfreundlichen Technologien produzieren, Industrien für die Herstellung hochwertiger Ausrüstungen, Biotechnologie, neue Energien, neue Materialien sowie Fahrzeuge, die mit neuen Energien angetrieben werden. Bereits im Beschluss des Staatsrats über die beschleunigte Entwicklung dieser Industrien („Decision on Accelerating the Development of Strategic Emerging Industries") vom Oktober 2010 wurde als Ziel vorgegeben, dass ihr Anteil am BIP auf $8 \%$ bis 2015 und 15\% bis 2020 erhöht werden soll (Schüller/Schüler-Zhou 2015).

Ein deutlicher Schwerpunkt der neuen Industriepolitik liegt Mei (2013) zufolge auf Nachhaltigkeit und Verbesserung traditioneller Industrien durch neue Technologien und Umweltschutz. Kritisch wird von Tan (2015) jedoch hervor- 
gehoben, dass eine Reihe bestehender Instrumente für die Durchsetzung einer stärker ökologisch orientierten Industriepolitik noch angepasst werden müsste. So würden hohe Subventionen für fossile Brennstoffe wie Kohle und Erdöl mit dem Ziel einer „Energiewende“ konfligieren. Von einer Reduzierung der Subventionen wären allerdings die großen im staatlichen Eigentum befindlichen energieintensiven Unternehmen betroffen, insbesondere in der Stahlindustrie, metallurgischen Industrie und Zementproduktion. Eine Subventionierung der Kohle- und Elektrizitätspreise für verarbeitende Industrieunternehmen - eine Praxis, die Lokalregierungen in ihrer Standortpolitik oft verfolgen - wäre dann auch nicht mehr möglich. In der Preispolitik bei fossilen Brennstoffen hat die Regierung allerdings versucht, im Sinne einer Energiewende erste Anpassungen durch eine höhere Besteuerung des privaten Benzinverbrauchs durchzusetzen.

Der Ausbau des Dienstleistungssektors sowie die Restrukturierung des verarbeitenden Gewerbes sind wichtige Felder der industriepolitischen Neuausrichtung. In seiner Rede vor dem Nationalen Volkskongress im Frühjahr 2015 kündigte Ministerpräsident Li Keqiang an, die verarbeitende Industrie durch eine innovationsgetriebene Entwicklungsstrategie zu einem Qualitätssprung bis zum Jahr 2025 zu führen. Im Rahmen eines zehnjährigen Aktionsplans (Made in China 2025) will die Regierung weitgehend unabhängig von ausländischer Technologie werden und nicht mehr nur „Werkstatt der Welt", sondern auch führend in neuen Industrien sein. Neben der IT-Industrie setzt die Regierung dabei auf Robotik, um steigende Löhne in China zu kompensieren und damit die preisliche Wettbewerbsfähigkeit gegenüber anderen Entwicklungsländern zu erhalten. Weiterhin soll die Produktion von Luftfahrt- und Raumfahrtausrüstungen gefördert werden. China will führend in der Satellitentechnik werden und eigene (große) Passagierflugzeuge bauen. Weitere Schwerpunkte sind die Herstellung von Ausrüstungen für Meerestechnik und hochentwickelten Halbleitern sowie Eisenbahnausrüstungen. Die Entwicklung dieser Industrien ist auch im $\mathrm{Zu}$ sammenhang mit der neuen außenwirtschaftlichen Expansionsstrategie Chinas zu sehen, die unter dem Namen „Silk Road“ (Seidenstraße)-Strategie oder „One Belt, One Road“ (Maritime Seidenstraße und Festland-Seidenstraße) bekannt ist. Hierbei geht es vor allem um den Ausbau der Verkehrsinfrastruktur entlang der Verkehrswege zwischen China und Europa zu Wasser und zu Land.

Im Aktionsplan „Made in China 2025“ sind ebenfalls Industrien mit energiesparenden Technologien und Fahrzeuge mit alternativen Antriebssystemen enthalten, durch die eine Verbesserung der Umweltbedingungen erzielt werden soll, aber die auch Chinas Position im globalen Automobilmarkt stärken sollen. Weiterhin ist die Förderung neuer Materialien vorgesehen sowie von Medikamenten, medizinischen Geräten und Landwirtschaftsmaschinen, durch die Chinas Abhängigkeit von ausländischen Produkten reduziert und die eigene Exportposition ausgeweitet werden soll (Deng 2015). Der ambitionierte Aktionsplan basiert auf einer Informatisierung der Fertigungstechnik mit dem Ziel der „smart factory“. Ähnlich wie in der deutschen Industrie-4.0-Strategie geht das chinesische Konzept von einer höheren Ressourceneffizienz und einer besseren Integration von Kunden und Geschäftspartnern aus, stellt jedoch hohe Anforderungen an die Automatisierung und Internetsicherheit. Zwar können sich durchaus Schnittstellen und Kooperationspotenziale für Deutschland und China in dem Bereich Industrie 4.0 ergeben, doch wird sich sicherlich auch der Wettbewerbsdruck für deutsche Unternehmen in China und weltweit erhöhen.

\section{Industriepolitische Steuerungs- probleme und Lerneffekte für andere Länder}

Chinas aktuelle Industriepolitik versucht, mit den Zielen des neuen Wirtschaftsmodells kompatibel zu sein. Große Herausforderungen bestehen vor allem bei der Nutzung energieeffizienter und umweltfreundlicher Technologien. Hier hat China in den vergangenen Jahrzehnten bereits einen enormen Technologiesprung vollzogen und seinen weltweiten Exportanteil bei Klimaschutzgütern, die zur Nutzung erneuerbarer Energie und zur Steigerung von Energieeffizienz eingesetzt werden, auf rund 20,1\% im Jahr 2011 (Deutschland 13,2\%) erhöhen können (Gehrke/Schasse 2013). Inzwischen kommen von den fünf weltweit führenden Solarherstellern mit der höchsten Photovoltaik-Produktion drei aus China. In vergleichbarer Weise haben sich die Marktanteile in der Windkraftindustrie verschoben. So entfiel im Jahr 2013 auf Chinas führenden Windkraftanlagenhersteller Goldwind Science \& Technology ein Marktanteil von $11 \%$. Nur Vestas Wind Systems, ein dänisches Unternehmen, konnte einen höheren Marktanteil (13,1\%) vorweisen. In Deutschland ist Goldwind vor allem durch seine Übernahme von Vensys im Jahr 2008 bekannt geworden. Der internationale Aufstieg von chinesischen Unternehmen in erneuerbaren Energien kann sowohl auf die starke Förderung des Staates zurückgeführt werden als auch auf ihre schnelle Internationalisierung mit Unternehmensaufkäufen, um technische Lücken zu schließen (Schüller/Schüler-Zhou 2015). Allerdings zeigt gerade das Beispiel staatlicher Förderung energieeffizienter Technologien die Schwächen der industriepolitischen „picking the winners"-Strategie in China. So begründen Kritiker die finanziellen Schwierigkeiten vieler Solarhersteller in China mit staatlicher Intervention und Subvention (Tan 2015).

Zwar steht die veränderte Rolle des Staates in der Ressourcenallokation im Mittelpunkt des 60-Punkte-Reformpakets von 2013, durch die die Unternehmen größere Entscheidungsrechte über Investition und Preisgestaltung erhalten sollen. Mehr Markt und die Beteiligung von Privatunternehmen sollen die dominante Stellung der Staatsunternehmen beschränken. Tatsächlich fand in den letzten Monaten jedoch eine Reihe zentralstaatlich gelenkter $\mathrm{Zu}$ - 
sammenschlüsse großer Staatsunternehmen statt. Ziel ist die Konsolidierung in bestimmten Industrien, um diese Unternehmen international wettbewerbsfähiger zu machen. So ist beispielsweise mit dem Zusammenschluss der China Southern Railways mit China Northern Railways ein Unternehmen entstanden, das größer als die bisherigen international aktiven Unternehmen wie Bombardier oder Siemens ist. Da bereits Verhandlungen mit 28 Ländern über den Verkauf von Schnellzügen laufen, ist die Zusammenlegung der beiden einzigen Konkurrenten aus Sicht der Zentralregierung sinnvoll, verstärkt jedoch ihre Marktmacht (Nikkei 2015). Während die Konsolidierung auch anderer Unternehmen beispielsweise in der Telekommunikation, im Schiffsbau und im Erdölsektor vorangetrieben wird, macht die Beteiligung privater Unternehmen nur wenig Fortschritte.

Lerneffekte der industriepolitischen Erfahrungen Chinas lassen sich nur sehr bedingt auf andere Länder übertragen. Im Vergleich zu westlichen Industrieländern sind die ordnungspolitischen Grundlagen und Beziehungsgeflechte zwischen den einzelnen Akteuren in China zu unterschiedlich. Für Länder mit nachholender Entwicklung bietet China jedoch ein Lernbeispiel für eine strategische und langfristig ausgerichtete Industriepolitik, die sich sowohl an der Wohlfahrt der Bevölkerung als auch an den Rahmenbedingungen für Wirtschaftswachstum wie Infrastruktur, Bildung und Gesundheit orientiert. Die Größe des Landes, seiner Bevölkerung und vor allem die hohe Zahl der arbeitsfähigen Bevölkerung zwingen die Regierung auch zu anderen Lösungen als sie in den westlichen Ländern praktikabel und sinnvoll sind. So ist trotz des allgemeinen Anstiegs der Produktionskosten in China eine Verlagerung von Industrien in andere kostengünstigere Länder nur bedingt eine Lösung. Die negativen Beschäftigungseffekte würden zu sozialen Unruhen in China führen und damit die Legitimität des politischen Systems infrage stellen. Beschäftigungsintensive Bereiche wie die verarbeitende Industrie werden deshalb auch zukünftig stärker in China zu finden sein als in anderen Ländern.

\section{LITERATUR}

Amsden, A. (1989): Asia's next giant: South Korea and the late industrialization, New York/Oxford

China Daily (2013): Tackling overcapacity is top priority, 22. November (china.org.cn)

Chu, W. W. (2011): How the Chinese government promoted a global automobile industry, in: Industrial and Corporate Change 20 (5), S. 1235-1276 Covington \& Burling (2014): Measures and practices restraining foreign investment in China, Report prepared for the European Commission DirectorateGeneral forTrade

Deng, Y. Q. (2015): Leaping into the first echelon. China strives to rise globally through a massive manufacturing overhaul, in: Beijing Review, April, http://www.bjreview.com.cn/special/2015-04/20/content_684291.htm

Gehrke, B./Schasse, U. (2013): Position Deutschlands im Außenhandel mit Gütern zur Nutzung erneuerbarer Energien und zur Steigerung der Energieeffizienz: Niedersächsisches Institut für Wirtschaftsforschung, Studien zum deutschen Innovationssystem (3), Hannover

Hall, P. A./Gingerich, D. W. (2004): Varieties of capitalism and institutional complementarities in the macroeconomy, MPIfG Discussion Paper 04/5 Hall, P. A./Soskice, D. (2001): Varieties of capitalism: the institutional foundations of comparative advantage, Oxford

Heilmann, S./Shih, L. (2013): The rise of industrial policy in China, 1978-2012, China Analysis (100), January, www.chinapolitik.de

Johnson, C. (1982): MITI and the Japanese miracle: the growth of industrial policy, 1925-75, Stanford

Knight, J. (2012): China as a developmental state, China Growth Center (CGC at St Edmund Hall: University of Oxford, CGC Discussion Paper Series (14), September
Lee, K./Cho, S.-J./Jin, J. (2009): Dynamics of catch-up in mobile phones and automobiles in China: sectoral systems of innovation perspectives, in: China Economic Journal (2) 1, S. 28-30

Liu, L. (2005): China's industrial policies and the global business revolution. The case of the domestic appliance industry, London/New York

Mei, J.T. (2013): How Europe should view China's industrial policy, http://europesworld.org/2013/10/01/how-europe-should-view-chinas-industrialpolicy/\#.VWnf40a_B1Y

Morrison, W. (2009): China and the global financial crisis: implications for the United States, Congressional Research Service, https://www.fas.org/sgp/crs/ row/RS22984.pdf

Naughton, B. (2007): The Chinese economy. Transition and growth, Boston Naughton, B. (2015): Is there a 'Xi Model' of economic reform? Acceleration of economic reform since Fall 2014, China Leadership Monitor (46) Nikkei, Asian Review (2015): China train giant gerger: CSR, and China CNR want the world, http://asia.nikkei.com/Business/Companies/CSR-China-CNRwant-the-world

PEW Research Center (2013): Environmental concerns on the rise in China, September 19, http://www.pewglobal.org/2013/09/19/environmental-concernson-the-rise-in-china/

Schucher, G. (2011): „Unausgeglichen, unkoordiniert, nicht nachhaltig“ Chinas Entwicklung vor großen Problemen, Focus Asien 3/2011 Schüller, M. (2008): Technologietransfer nach China - Ein unkalkulierbares Risiko für die Länder derTriade USA, Europa und Japan?, Berlin Schüller, M. (2009): China in der globalen Finanzmarktkrise: Wirtschaftspolitische Strategien und Strukturprobleme, Focus Asien 4/2009

Schüller, M. (2012): Industriepolitik in den BRIC-Staaten, in: Allespach, M./Ziegler, A. (Hrsg.): Zukunft des Industriestandortes Deutschland 2020, Marburg, S. 302-320

Schüller, M./Conlé, M. (2007): Indien und China auf der technologischen Überholspur?, in: Orientierungen zur Wirtschafts- und Gesellschaftspolitik 113/2007, S. $61-67$

Schüller, M./Schüler-Zhou, Y. (2015): China: Die neue Innovationssupermacht? Asien Focus 1/2015

Tan, H. (2015): Greening China:Tackling bad industrial policies should be a priority: Institute of Development Studies, http://www.ids.ac.uk/opinion/ greening-china-tackling-bad-industrial-policies-should-be-a-priority ten Brink, T. (2013): Wirtschaftsregulierung in China, in: dms - der moderne staat - Zeitschrift für Public Policy, Recht und Management 6 (1), S. 65-84 UNIDO (United Nations Industrial and Development Organization) (2013): Industrial development report, sustaining employment growth: the role of manufacturing and structual change, November

Wade, R. (1990): Governing the market: economic theory and the role of government in East Asina industrialization, Princeton

Wade, R. (2003): What strategies are viable for developing countries today? The World Trade Organization and the shrinking of 'developmental space': Crisis States Research Centre Working Papers Series (31)

Walsh, K. (2003): Foreign high-tech R\&D in China. Risks, rewards, and implications for U.S. - China relations: The Henry L. Stimson Center, Washington

Wogart, J.-P./Schüller, M. (2011):The EU's Blue Card: Will it attract Asia's highly skilled? GIGA Focus International Edition (3)

Vissers, G./Dankbaar, B. (2013): Path dependence and path plasticity: Textile cities in the Netherlands, in: Zeitschrift für Wirtschaftsgeographie 57 (1-2), S. 83-95

Winkel, A. (2012): Wirtschaftspolitik in China und Indien im Vergleich. Arbeitspapiere der Arbeitsstelle Internationale Politische Ökonomie 9/2012 Yergin, D./Stanislaw, J. (1998): The commanding heights, New York

\section{AUTORIN}

MARGOT SCHÜLLER, Dr., ist Wissenschaftliche Mitarbeiterin am German Institute of Global and Area Studies (GIGA), Institut für Asien-Studien in Hamburg. Arbeitsschwerpunkte: Analyse der wirtschaftlichen Entwicklung in China, insbesondere der Industriepolitik sowie der Internationalisierung chinesischer Unternehmen.

margot.schueller@giga-hamburg.de 